\title{
Metallosupramolecular Coordination Complexes: The Design of Heterometallic 3d-4f Gridlike Structures
}

Jianfeng Wu, Lang Zhao, Li Zhang, Xiao-Lei Li, Mei Guo and Jinkui Tang*

Table S1. Crystallographic data for complexes 1-3.

\begin{tabular}{|c|c|c|c|}
\hline & 1 & 2 & 3 \\
\hline Formula & $\mathrm{C}_{88} \mathrm{H}_{92} \mathrm{Dy}_{4} \mathrm{~N}_{32} \mathrm{O}_{32} \mathrm{~S}_{4} \mathrm{Zn}_{4}$ & $\mathrm{C}_{96} \mathrm{H}_{112} \mathrm{Co}_{3} \mathrm{Dy}_{4} \mathrm{~N}_{38} \mathrm{O}_{36} \mathrm{~S}_{10}$ & $\mathrm{C}_{92} \mathrm{H}_{104} \mathrm{Cl}_{2} \mathrm{Cu}_{5} \mathrm{Dy}_{4} \mathrm{~N}_{36} \mathrm{O}_{36} \mathrm{~S}_{8}$ \\
\hline $\mathrm{FW}, \mathrm{g} \cdot \mathrm{mol}^{-1}$ & 3149.65 & 3521.63 & 3585.19 \\
\hline crystal system & Tetragonal & Orthorhombic & Tetragonal \\
\hline space group & $I 4(1) / a$ & Pmmn & $I 4(1) / a$ \\
\hline$T, \mathrm{~K}$ & $273(2)$ & $296(2)$ & $296(2)$ \\
\hline$\lambda, \AA$ & 0.71073 & 0.71073 & 0.71073 \\
\hline$a, \AA$ & $24.0282(16)$ & 19.507(2) & 23.205(3) \\
\hline$b, \AA$ & $24.0282(16)$ & 26.831(3) & 23.205(3) \\
\hline$c, \AA$ & $34.364(2)$ & $16.223(2)$ & $34.382(5)$ \\
\hline$\alpha,{ }^{\circ}$ & 90 & 90 & 90 \\
\hline$\beta, \circ$ & 90 & 90 & 90 \\
\hline$\gamma, \circ$ & 90 & 90 & 90 \\
\hline$V, \AA^{3}$ & $19840(2)$ & 8491.3(18) & $18514(4)$ \\
\hline $\mathrm{Z}$ & 4 & 2 & 4 \\
\hline$\rho_{\text {calcd }}, \mathrm{g} \cdot \mathrm{cm}^{-3}$ & 1.054 & 1.377 & 1.286 \\
\hline GOF on $F^{2}$ & 1.079 & 1.144 & 1.116 \\
\hline reflns collected & 47083 & 52179 & 56258 \\
\hline$R_{1}^{a)}, w R_{2}(\mathrm{I} \geq 2 \sigma(I))^{b)}$ & $0.0914,0.2818$ & $0.0963,0.2613$ & $0.0705,0.2131$ \\
\hline$R_{1}, w R_{2}$ (all data) & $0.1415,0.3125$ & $0.1266,0.2882$ & $0.0987,0.2438$ \\
\hline CCDC number & 1418805 & 1418806 & 1418807 \\
\hline
\end{tabular}

${ }^{a)} R_{1}=\sum|| F_{\mathrm{o}}|-| F_{\mathrm{c}}|| \sum\left|F_{\mathrm{o}}\right|,{ }^{b)} w R_{2}=\left[\sum w\left(F_{\mathrm{o}}{ }^{2}-F_{\mathrm{c}}{ }^{2}\right)^{2} / \sum w\left(F_{\mathrm{o}}{ }^{2}\right)^{2}\right]^{1 / 2}$ 
Table S2. Selected bond distances ( $\AA$ ) for complexes 1-3.

\begin{tabular}{|c|c|c|c|c|c|}
\hline \multicolumn{2}{|c|}{ Complex 1} & \multicolumn{2}{|c|}{ Complex 2} & \multicolumn{2}{|c|}{ Complex 3} \\
\hline Dy(1)-O(5) & $2.428(10)$ & $\mathrm{Dy}(1)-\mathrm{O}(3)$ & $2.346(7)$ & $\mathrm{Dy}(1)-\mathrm{O}(2)$ & $2.385(7)$ \\
\hline $\mathrm{Dy}(1)-\mathrm{O}(2)$ & $2.340(9)$ & Dy(1)-O(5) & $2.378(9)$ & $\mathrm{Dy}(1)-\mathrm{O}(4)$ & $2.415(7)$ \\
\hline Dy(1)-O(3)\#1 & $2.387(9)$ & Dy(1)-O(1) & $2.387(8)$ & Dy(1)-O(1) & $2.458(7)$ \\
\hline Dy(1)-O(1) & $2.418(10)$ & Dy(1)-N(7) & $2.454(9)$ & Dy(1)-N(7) & $2.476(8)$ \\
\hline $\mathrm{Dy}(1)-\mathrm{O}(4)$ & $2.414(10)$ & $\mathrm{Dy}(1)-\mathrm{O}(2)$ & $2.455(8)$ & Dy(1)-N(1) & $2.490(7)$ \\
\hline Dy(1)-N(2) & $2.447(11)$ & Dy(1)-O(4) & 2.463(9) & Dy(1)-N(6) & $2.514(8)$ \\
\hline Dy(1)-N(6) & $2.467(11)$ & Dy(1)-N(1) & $2.464(10)$ & Dy(1)-N(2) & 2.525(8) \\
\hline Dy(1)-N(7) & $2.485(10)$ & Dy(1)-N(2) & $2.491(10)$ & Dy(1)-O(3) & $2.375(6)$ \\
\hline Dy(1)-N(1) & $2.518(10)$ & Dy(1)-N(8) & $2.515(10)$ & $\mathrm{Cu}(1)-\mathrm{N}(4)$ & $1.947(8)$ \\
\hline Zn(1)-N(3) & $2.168(12)$ & Co(1)-N(9) & $2.063(18)$ & $\mathrm{Cu}(1)-\mathrm{N}(8)$ & $1.972(9)$ \\
\hline Zn(1)-N(4) & $2.049(9)$ & $\mathrm{Co}(1)-\mathrm{N}(10)$ & $2.067(16)$ & $\mathrm{Cu}(1)-\mathrm{N}(3)$ & $2.075(8)$ \\
\hline $\mathrm{Zn}(1)-\mathrm{N}(5)$ & $2.176(11)$ & Co(1)-N(5) & $2.087(11)$ & $\mathrm{Cu}(1)-\mathrm{N}(5)$ & $2.085(8)$ \\
\hline Zn(1)-N(8) & $1.969(12)$ & $\mathrm{Co}(1)-\mathrm{N}(6)$ & $2.174(10)$ & $\mathrm{Cu}(1)-\mathrm{S}(1)$ & $2.606(3)$ \\
\hline $\mathrm{Zn}(1)-\mathrm{O}(6)$ & $1.956(13)$ & Co(1)-S(3) & $2.629(6)$ & $\mathrm{Cu}(2)-\mathrm{N}(9)$ & $2.027(9)$ \\
\hline Dy(1)-Zn(1) & $5.5164(16)$ & $\mathrm{Co}(3)-\mathrm{N}(12)$ & $1.97(2)$ & Dy(1)-Cu(1) & $5.4851(14)$ \\
\hline \multirow[t]{3}{*}{$\operatorname{Zn}(1)-Z n(1) \# 1$} & 11.8385(22) & $\mathrm{Co}(3)-\mathrm{N}(11)$ & 1.989(19) & $\mathrm{Dy}(1)-\mathrm{Cu}(2)$ & 7.6363(9) \\
\hline & & Dy(1)-Co(1) & 5.4998(9) & $\mathrm{Cu}(1)-\mathrm{Cu}(2)$ & $5.7401(12)$ \\
\hline & & Dy(1)-Co(3) & 7.7193(8) & $\mathrm{Cu}(1)-\mathrm{Cu}(1) \# 1$ & 8.1933(17) \\
\hline \multicolumn{2}{|c|}{$\# 1 \mathrm{y}+1 / 4,-\mathrm{x}+7 / 4,-\mathrm{z}+3 / 4$} & Co(1)-Co(3) & $6.0655(27)$ & \multicolumn{2}{|c|}{$\# 1$ y-1/4,-x+5/4,-z+1/4 } \\
\hline
\end{tabular}

Table S3. The CShM values calculated by SHAPE $2.1^{1}$ for 1-3.

\begin{tabular}{c|cccc}
\hline Central atom & Coordination Geometry & Complex 1 & Complex 2 & Complex 3 \\
\hline \multirow{2}{*}{ Dy } & Capped square antiprism J10 $\left(C_{4 v}\right)$ & 2.532 & 3.044 & 2.106 \\
& Spherical capped square antiprism $\left(C_{4 v}\right)$ & 1.622 & 2.133 & 1.400 \\
& Tricappedtrigonal prism J51 $\left(D_{3 h}\right)$ & 4.331 & 4.730 & 3.753 \\
& Spherical tricappedtrigonal prism $\left(D_{3 h}\right)$ & 1.907 & 2.009 & 1.837 \\
\hline
\end{tabular}




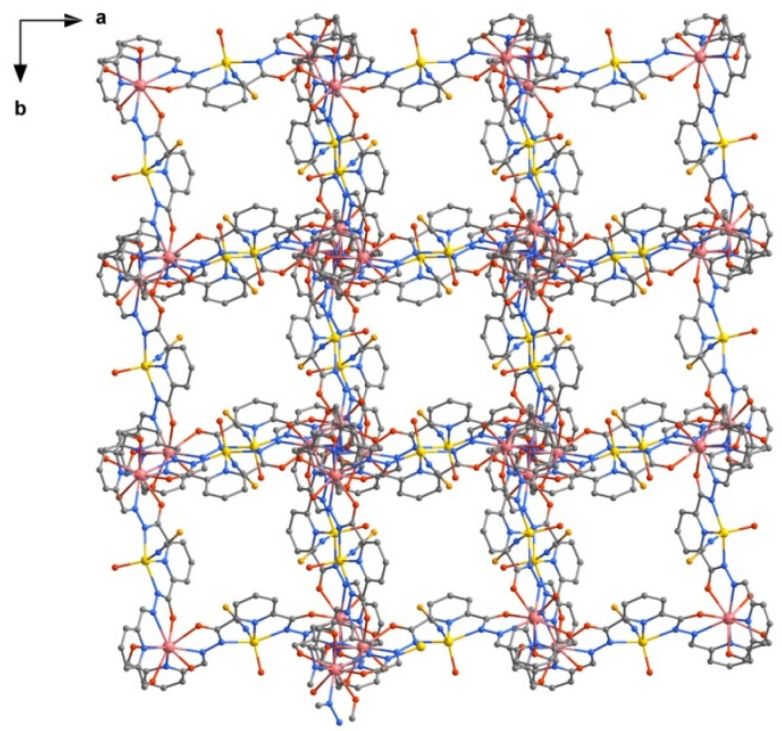

Figure S1. Packing model of complex $\mathbf{1}$ along $c$ axis; solvents have been omitted for clarity.

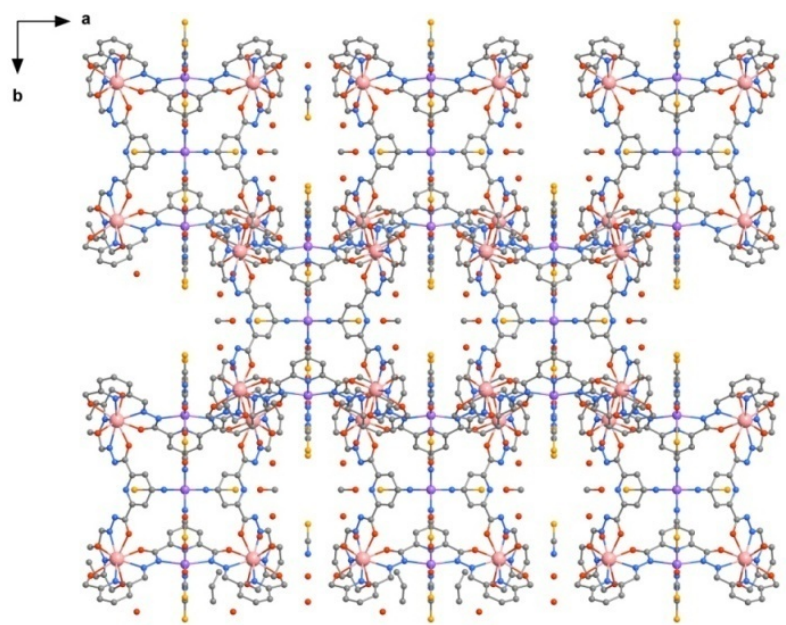

Figure S2. Packing model of complex 2 along $c$ axis.

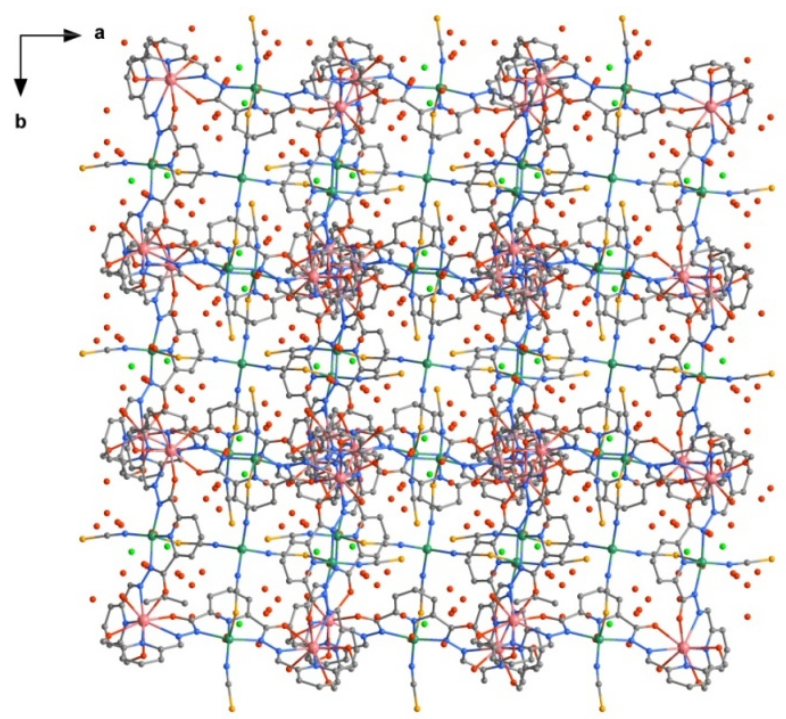

Figure S3. Packing model of complex 3 along $c$ axis. 


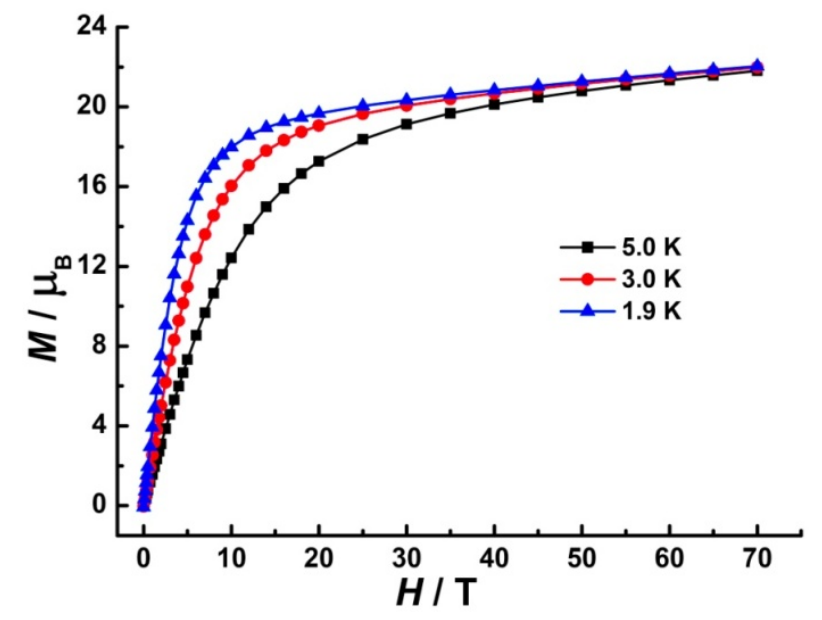

Figure S4. Molar magnetization $(M)$ vs. magnetic field $(H)$ for $\mathbf{1}$ at 1.9, 3.0, and $5.0 \mathrm{~K}$.

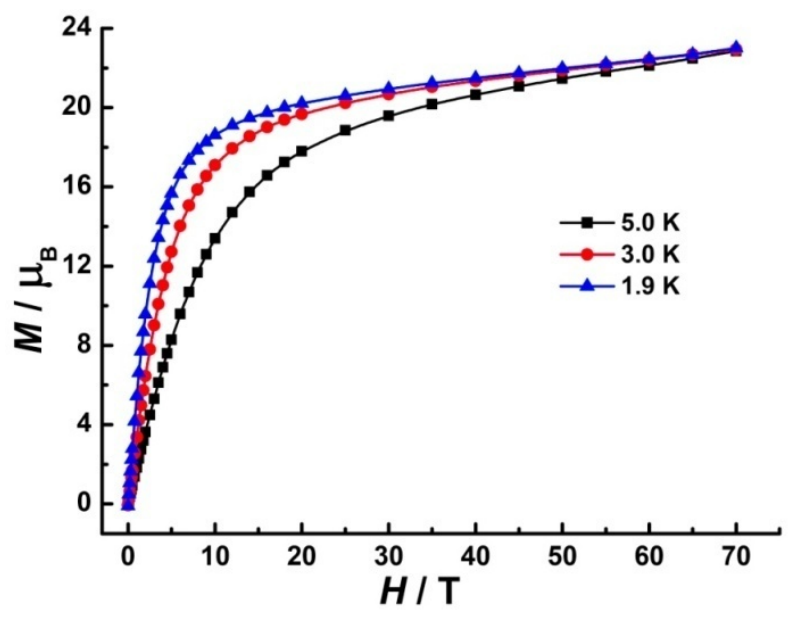

Figure S5. Molar magnetization $(M)$ vs. magnetic field $(H)$ for 2 at 1.9, 3.0, and $5.0 \mathrm{~K}$.

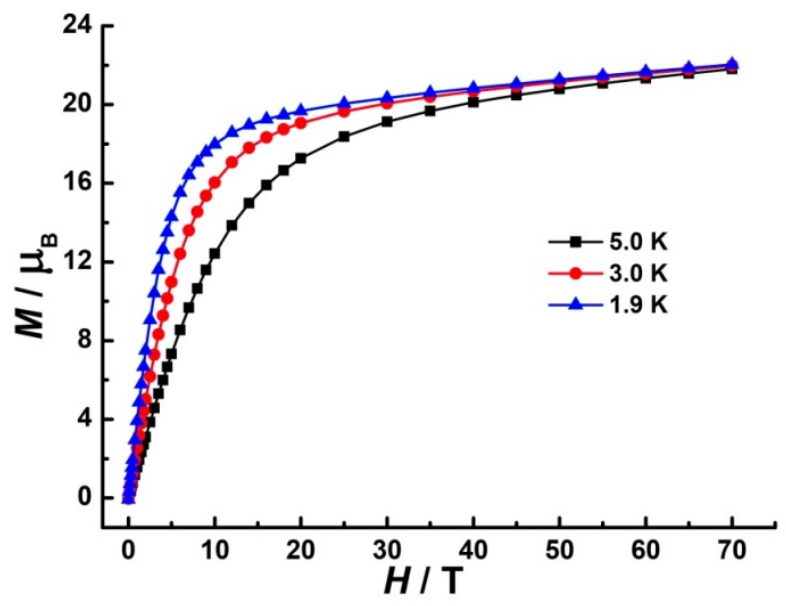

Figure S6. Molar magnetization $(M)$ vs. magnetic field $(H)$ for 3 at 1.9, 3.0, and $5.0 \mathrm{~K}$. 


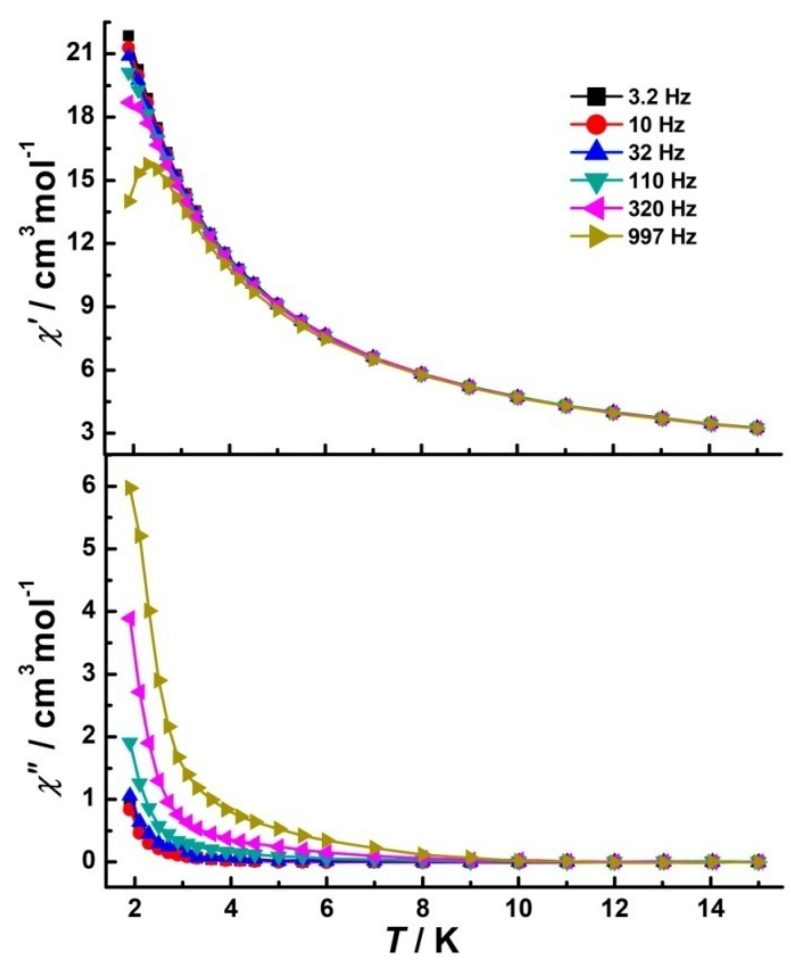

Figure S7. Temperature-dependent ac susceptibility for $\mathbf{1}$ at indicated frequencies, under 500 Oe dc field.

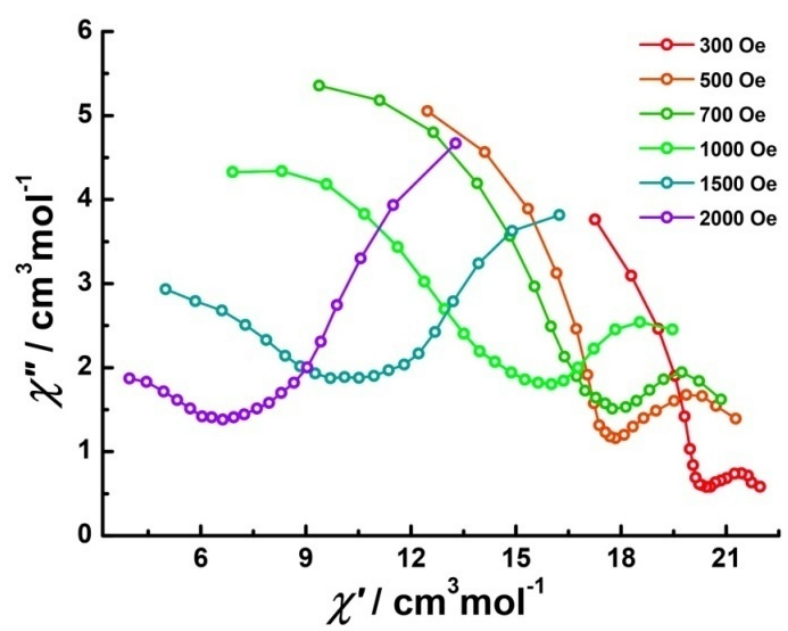

Figure S8. Cole-Cole plots for $\mathbf{1}$ under indicated dc field at $2 \mathrm{~K}$. The solid lines are guides for the eyes. 


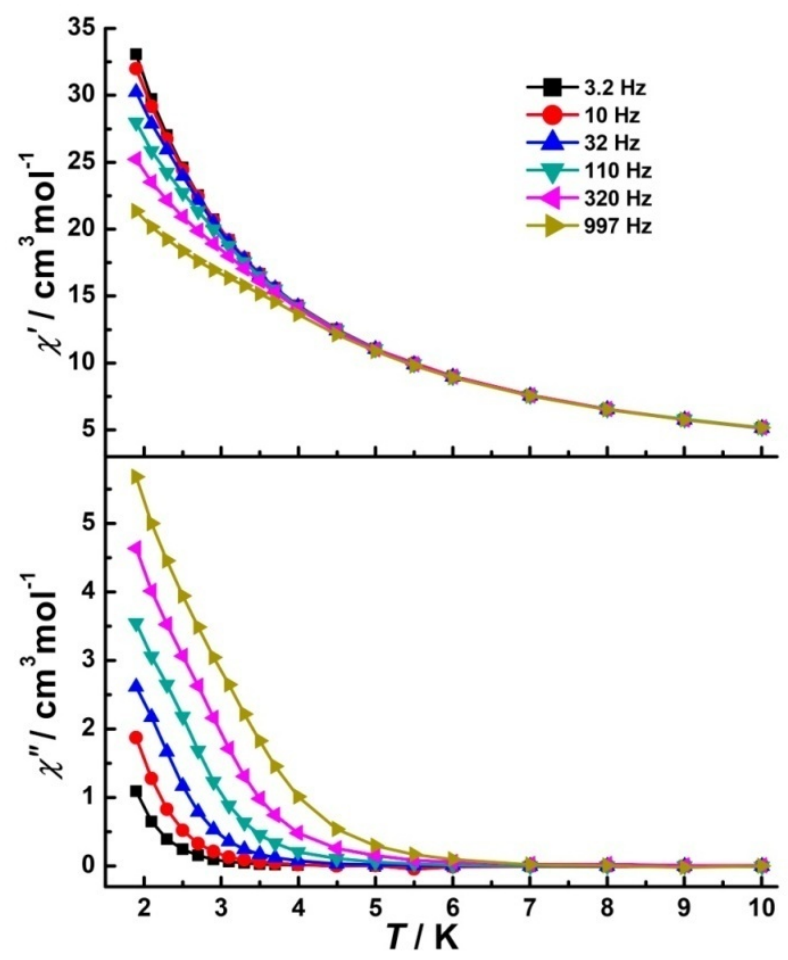

Figure S9. Temperature-dependent ac susceptibility for $\mathbf{2}$ at indicated frequencies, under zero dc field.

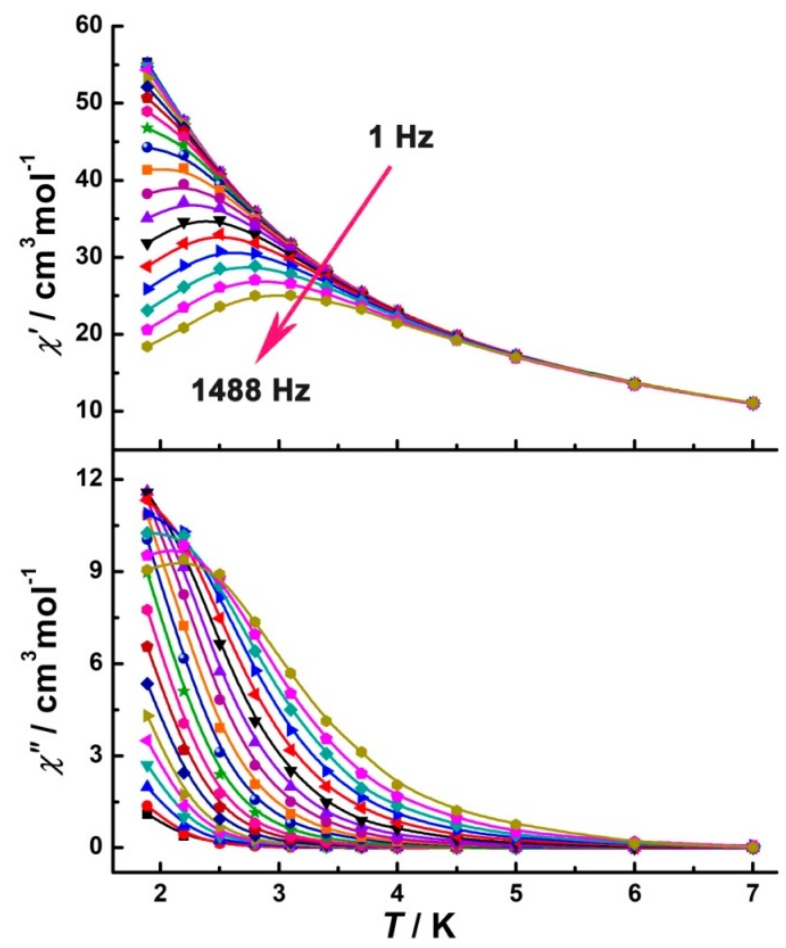

Figure S10.Temperature-dependent ac susceptibility for $\mathbf{3}$ at indicated frequencies, under zero dc field. 


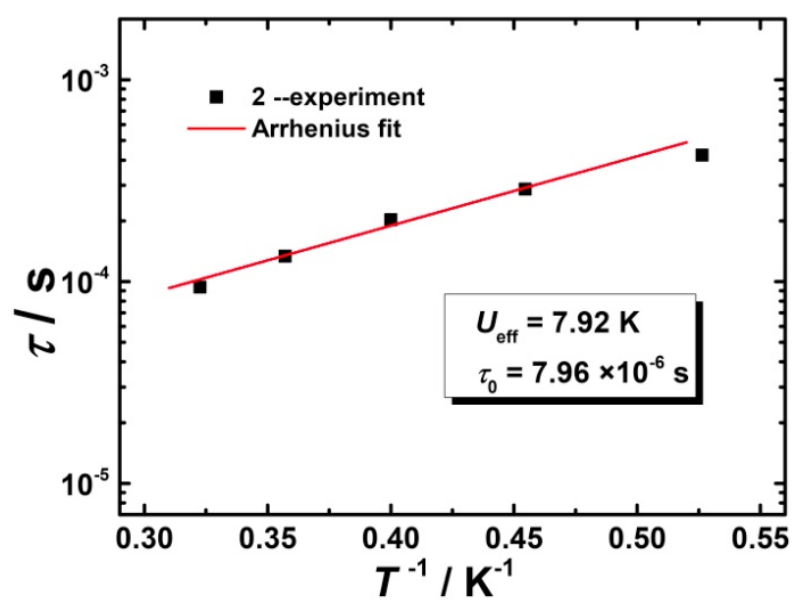

Figure S11. Plot of $\tau$ vs. $T^{1}$ for 2 , obtained under zero dc fields over the temperature range 1.9-3.1 K. The red line represents the Arrhenius fitted result.

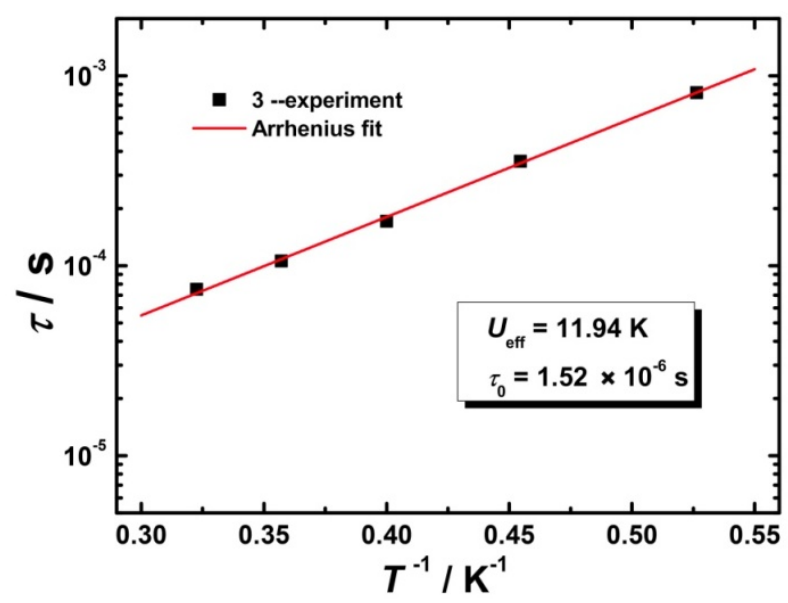

Figure S12. Plot of $\tau$ vs. $T^{1}$ for 3, obtained under zero dc fields over the temperature range 1.9-3.1 K. The red line represents the Arrhenius fitted result.

1. Casanova, D.; Alemany, P.; Bofill, J. M.; Alvarez, S., Chem. Eur. J. 2003, 9, 1281. 